TRANSACTIONS OF THE

AMERICAN MATHEMATICAL SOCIETY

Volume 361, Number 5, May 2009, Pages 2679-2688

S 0002-9947(08)04629-1

Article electronically published on November 18, 2008

\title{
CONGRUENCE FOR RATIONAL POINTS OVER FINITE FIELDS AND CONIVEAU OVER LOCAL FIELDS
}

\author{
HÉLÈNE ESNAULT AND CHENYANG XU
}

\begin{abstract}
If the $\ell$-adic cohomology of a projective smooth variety, defined over a local field $K$ with finite residue field $k$, is supported in codimension $\geq 1$, then every model over the ring of integers of $K$ has a $k$-rational point. For $K$ a $p$-adic field, this is proved in (Esnault, 2007, Theorem 1.1). If the model $\mathcal{X}$ is regular, one has a congruence $|\mathcal{X}(k)| \equiv 1$ modulo $|k|$ for the number of $k$-rational points (Esnault, 2006, Theorem 1.1). The congruence is violated if one drops the regularity assumption.
\end{abstract}

\section{INTRODUCTION}

Let $X$ be a projective variety defined over a local field $K$ with finite residue field $k=\mathbb{F}_{q}$. Let $R$ be the ring of integers of $K$. A model of $X / K$ is a flat projective morphism $\mathcal{X} \rightarrow \operatorname{Spec}(R)$, with $\mathcal{X}$ an integral scheme, such that tensored with $K$ over $R$, it coincides with $X \rightarrow \operatorname{Spec}(K)$. As in 9 and 10, we consider $\ell$-adic cohomology $H^{i}(\bar{X})$ with $\mathbb{Q}_{\ell}$-coefficents. Recall briefly that one defines the first coniveau level

$$
N^{1} H^{i}(\bar{X})=\left\{\alpha \in H^{i}(\bar{X}), \exists \text { divisor } D \subset X \text { s.t. } 0=\left.\alpha\right|_{X \backslash D} \in H^{i}(\overline{X \backslash D})\right\} .
$$

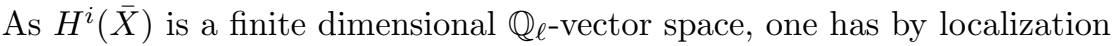

$$
\exists D \subset X \text { s.t. } N^{1} H^{i}(\bar{X})=\operatorname{Im}\left(H_{\bar{D}}^{i}(\bar{X}) \rightarrow H^{i}(\bar{X})\right),
$$

where $D \subset X$ is a divisor. One says that $H^{i}(\bar{X})$ is supported in codimension $\geq 1$ if $N^{1} H^{i}(\bar{X})=H^{i}(\bar{X})$. The purpose of this note is twofold. We show the following theorem.

Theorem 1.1. Let $X$ be a smooth, projective, absolutely irreducible variety defined over a local field $K$ with finite residue field $k$. Assume that $\ell$-adic cohomology $H^{i}(\bar{X})$ is supported in codimension $\geq 1$ for all $i \geq 1$. Let $\mathcal{X}$ be a model of $X$ over the ring of integers $R$ of $K$. Then there is a projective surjective morphism $\sigma: \mathcal{Y} \rightarrow \mathcal{X}$ of $R$-schemes such that

$$
|\mathcal{Y}(k)| \equiv 1 \bmod |k| .
$$

In particular, any model $\mathcal{X} / R$ of $X / K$ has a $k$-rational point.

Received by the editors June 7, 2007 and, in revised form, August 27, 2007.

2000 Mathematics Subject Classification. Primary 14G15, 14G05.

Key words and phrases. Rational point, congruence, coniveau.

This work was partially supported by the DFG Leibniz Preis and the American Institute for Mathematics.

(C)2008 American Mathematical Society Reverts to public domain 28 years from publication 
This generalizes [10, Theorem 1.1] where the theorem is proven under the assumption that $K$ has characteristic 0 . On the other hand, assuming that $\mathcal{X}$ is regular, we showed in [9, Theorem 1.1] that the number of $k$-rational points $|\mathcal{X}(k)|$ is congruent to 1 modulo $|k|$. It was in fact the way to show that $k$-rational points exist on $\mathcal{X}$, as surely $|k|$, being a $p$-power, where $p$ is the characteristic of $k$, is $>1$. We show that if we drop the regularity assumption, there are models which, according to Theorem 1.1 have a rational point, but do not satisfy the congruence.

Theorem 1.2. Let $X_{0}=\mathbb{P}^{2}$ over $K_{0}:=\mathbb{Q}_{p}$ or $\mathbb{F}_{p}((t))$. Then there is a finite field extension $K \supset K_{0}$, which can be chosen to be unramified, and there is a normal model $\mathcal{X} / R$ of $X:=X_{0} \otimes_{K_{0}} K$, such that $|\mathcal{X}(k)|$ is not congruent to 1 modulo $|k|$.

The $\ell$-adic proof of Theorem 1.1 closely follows the one of unequal characteristic in [10. Theorem 1.1], and, in addition to Deligne's integrality theorem [7, Corollaire 5.5.3] and [9, Appendix] and purity [11], relies strongly on de Jong's alteration theorem as expressed in [6]. However, we have to replace the trace argument we used there by a more careful analysis of the Leray spectral sequence stemming from de Jong's construction. The construction of the examples in Theorem 1.2 uses Artin's contraction theorem as expressed in [1 and is somewhat inspired by Kollár's construction exposed in 4, Section 3.3].

\section{Proof of Theorem 1.1}

This section is devoted to the proof of Theorem 1.1

Let $K$ be a local field with finite residue field $k$. Let $R \subset K$ be its valuation ring. Let $\mathcal{X} \rightarrow \operatorname{Spec} R$ be a model of a projective variety $X \rightarrow \operatorname{Spec} K$. We do not assume here that $X$ is absolutely irreducible, nor do we assume that $X / K$ is smooth. Then by [6, Corollary 5.15], there is a diagram

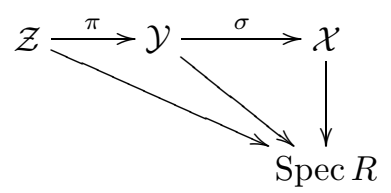

and a finite group $G$ acting on $\mathcal{Z}$ over $\mathcal{Y}$ with the properties

(i) $\mathcal{Z} \rightarrow \operatorname{Spec} R$ and $\mathcal{Y} \rightarrow \operatorname{Spec} R$ are flat,

(ii) $\sigma$ is projective, surjective, $K(\mathcal{X}) \subset K(\mathcal{Y})$ is a purely inseparable field extension,

(iii) $\mathcal{Y}$ is the quotient of $\mathcal{Z}$ by $G$,

(iv) $\mathcal{Z}$ is regular.

We want to show that this $\mathcal{Y}$ is a model satisfying the congruence $|\mathcal{Y}(k)| \equiv 1$ modulo $|k|$ of Theorem 1.1. Let us set

$$
Y=\mathcal{Y} \otimes K, Z=\mathcal{Z} \otimes K
$$

The only difference from [10, (2.1)] is that $K(\mathcal{X}) \subset K(\mathcal{Y})$ may be a purely inseparable extension rather than an isomorphism. Thus, the argument there breaks down, as one does not have traces as in [10, (2.3), (2.4)]. We do not have [10, (2.5)] a priori, and we cannot conclude [10, Claim 2.1].

Let us overtake the notations of loc. cit.: we endow all schemes considered (which are $R$-schemes) with the upper subscript ${ }^{u}$ to indicate the base change $\otimes_{R} R^{u}$ or $\otimes_{K} K^{u}$, where $K^{u} \supset K$ is the maximal unramified extension and $R^{u} \supset R$ 
is the normalization of $R$ in $K^{u}$. Likewise, we write $\bar{?}$ to indicate the base change $\otimes_{R} \bar{R}, \otimes_{K} \bar{K}, \otimes_{k} \bar{k}$, where $\bar{K} \supset K, \bar{k} \supset k$ are the algebraic closures and $\bar{R} \supset R$ is the normalization of $R$ in $\bar{K}$. We consider as in [9, (2.1)] the $F$-equivariant exact sequence $([8,3.6(6)])$

$$
\ldots \rightarrow H_{\bar{B}}^{i}\left(\mathcal{Y}^{u}\right) \stackrel{\iota}{\rightarrow} H^{i}(\bar{B})=H^{i}\left(\mathcal{Y}^{u}\right) \stackrel{s p^{u}}{\longrightarrow} H^{i}\left(Y^{u}\right) \rightarrow \ldots,
$$

where $F \in \operatorname{Gal}(\bar{k} / k)$ is the geometric Frobenius, and $B=\mathcal{Y} \otimes k$. We have [10, Claim 2.2] unchanged:

Claim 2.1. The eigenvalues of the geometric Frobenius $F \in \operatorname{Gal}(\bar{k} / k)$ acting on $H_{\bar{B}}^{i}\left(\mathcal{Y}^{u}\right)$, thus a fortiori on $\iota\left(H_{\bar{B}}^{i}\left(\mathcal{Y}^{u}\right)\right) \subset H^{i}(\bar{B})$, lie in $q \cdot \overline{\mathbb{Z}}$ for all $i \geq 1$.

Proof. For sake of completeness, we reproduce the proof of [9, Theorem 2.2], which is itself derived from [10, Claim 2.2]. By (iii), one has $H_{\bar{B}}^{i}\left(\mathcal{Y}^{u}\right)=H_{\bar{C}}^{i}\left(\mathcal{Z}^{u}\right)^{G} \subset$ $H_{\bar{C}}^{i}\left(\mathcal{Z}^{u}\right)$, where $C=\pi^{-1}(B)$. By (iv), $\mathcal{Z}$ is regular. Thus $\mathcal{Z}^{u}$, being the base change of $\mathcal{Z}$ by the unramified extension $R^{u} \supset R$, is regular as well. So it is enough to show that the eigenvalues of $F$ acting on $H_{\bar{C}}^{i}\left(\mathcal{Z}^{u}\right)$ lie in $q \cdot \overline{\mathbb{Z}}$ for all $i \geq 1$, where now the scheme $\mathcal{Z}^{u}$ is regular and $C$ has codimension $\geq 1$. Let $C^{0} \subset C$ be the smooth locus of $C$, let $C^{1} \subset C \backslash C^{0}$ be the smooth locus of $C^{0}$, etc. Then $\bar{C}^{i}$ is smooth. Using localization

$$
\ldots \rightarrow H_{\bar{C}^{1}}^{i}\left(\mathcal{Z}^{u}\right) \rightarrow H_{\bar{C}}^{i}\left(\mathcal{Z}^{u}\right) \rightarrow H_{\bar{C}^{0}}^{i}\left(\mathcal{Z}^{u} \backslash \bar{C}^{1}\right) \rightarrow \ldots
$$

and purity $H^{i-2}\left(\bar{C}^{0}\right)(-1) \cong H_{\bar{C}^{0}}^{i}\left(\mathcal{Z}^{u} \backslash \bar{C}^{1}\right)$ ([11, Theorem 2.1.1]), etc., one reduces the problem to integrality of the eigenvalues of $F$ acting on $H^{j}(\bar{D})$ for any smooth variety $D$ defined over $k$ and any $j \geq 1$. One then applies Deligne's integrality theorem [7, Lemme 5.5.3 iii)] and duality on $D$ or directly [9, Appendix, Corollary 0.4$]$.

So the problem is to show that the eigenvalues of $F$ acting on $\operatorname{Im}\left(s p^{u}\right) \subset H^{i}\left(Y^{u}\right)$ lie in $q \cdot \overline{\mathbb{Z}}$ as well. One has the following claim.

Claim 2.2. The eigenvalues of the geometric Frobenius $F \in \operatorname{Gal}(\bar{k} / k)$ acting on $H^{i}\left(Y^{u}\right)$, and therefore on $\operatorname{Im}\left(s p^{u}\right) \subset H^{i}\left(Y^{u}\right)$, lie in $q \cdot \overline{\mathbb{Z}}$ for all $i \geq 1$.

Proof. Let us decompose the morphism $\sigma$ as

$$
\sigma: Y \stackrel{\tau}{\rightarrow} X_{1} \stackrel{\epsilon}{\rightarrow} X
$$

where $X_{1}$ is the normalization of $X$ in $K(Y)$. Thus in particular, $\tau$ is birational, and $\epsilon$ is finite and purely inseparable. Let us denote by $U \subset X$ a non-empty open set such that $\left.\tau\right|_{\epsilon^{-1}(U)}: \tau^{-1} \epsilon^{-1}(U) \rightarrow \epsilon^{-1}(U)$ is an isomorphism, and let us set $D:=X \backslash U$. We define

$$
\mathcal{C}:=\operatorname{cone}\left(\mathbb{Q}_{\ell} \rightarrow R \tau_{*} \mathbb{Q}_{\ell}\right)[-1]
$$

as an object in the bounded derived category of $\mathbb{Q}_{\ell}$-constructible sheaves on $X_{1}$. Since $\tau_{*} \mathbb{Q}_{\ell}=\mathbb{Q}_{\ell}$, the cohomology sheaves of $\mathcal{C}$ are in degree $\geq 1$, and have support in $D_{1}:=D \times_{X} X_{1}$. We conclude

$$
H_{D_{1}^{u}}^{i}\left(X_{1}^{u}, \mathcal{C}\right)=H^{i}\left(X_{1}^{u}, \mathcal{C}\right) \forall i \geq 0
$$


One has the commutative diagram of exact sequences

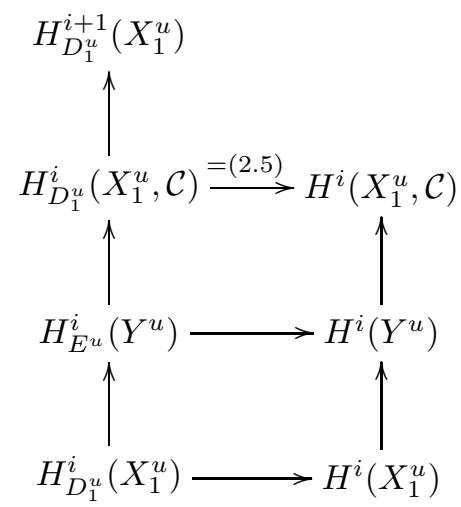

where $E=\sigma^{-1}(D)$. So to show the claim, via the right vertical exact sequence, it is enough to show that the eigenvalues of $F$ acting on $H^{i}\left(X_{1}^{u}\right)$ and on $H^{i}\left(X_{1}^{u}, \mathcal{C}\right)$ lie in $q \cdot \overline{\mathbb{Z}}$. This is true on $H^{i}\left(X_{1}^{u}\right)$ by $\left[9\right.$, Theorem 1.5 and Appendix]. For $H^{i}\left(X_{1}^{u}, \mathcal{C}\right)$, via the left vertical exact sequence, it is enough to show that the eigenvalues of $F$ acting on $H_{E^{u}}^{i}\left(Y^{u}\right)$ and on $H_{D^{u}}^{i+1}\left(X_{1}^{u}\right)$ lie in $q \cdot \overline{\mathbb{Z}}$. Writing $H_{E^{u}}^{i}\left(Y^{u}\right)=H_{L^{u}}^{i}\left(Z^{u}\right)^{G}$ where $L=D \times_{X} Z$, one is reduced to showing that the eigenvalues of $F$ acting on $H_{V^{u}}^{j}\left(W^{u}\right)$ lie in $q \cdot \overline{\mathbb{Z}}$ for $W$ a regular $K$-scheme and $V \subset W$ a closed $K$-subscheme of codimension $c \geq 1$. If $V$ is regular, one applies purity $H^{j-2 c}\left(V^{u}\right)(-c) \cong H_{V^{u}}^{j}\left(W^{u}\right)$ again, and one is reduced to showing that the eigenvalues of $F$ acting on $H^{i}\left(V^{u}\right)$ lie in $\overline{\mathbb{Z}}$ for all $i \geq 0$. One applies [9, Appendix, Corollary 0.3]. If $V$ is not regular, one writes the $F$-equivariant exact sequence $\ldots \rightarrow H_{\left(V^{1}\right)^{u}}^{i}\left(W^{u}\right) \rightarrow H_{V^{u}}^{i}\left(W^{u}\right) \rightarrow$ $H_{\left(V^{0}\right)^{u}}^{i}\left(\left(W^{0}\right)^{u}\right) \rightarrow \ldots$, where $V^{0} \subset V$ is the regular locus, $W^{0}=W \backslash V^{1}, V^{1}=$ $V \backslash V^{0}$ and one argues inductively as in the proof of Claim 2.1.

We now conclude the proof of Theorem 1.1 all the eigenvalues of $F$ acting on $H^{i}(\bar{B})$ lie in $q \cdot \overline{\mathbb{Z}}$ for $i \geq 1$; thus the Grothendieck-Lefschetz trace formula applied to $H^{*}(\bar{B})$, together with the absolute connectedness of $B$, which follows from the absolute irreducibility of $Y$, imply the congruence. This finishes the proof of Theorem 1.1. To summarize: $\mathcal{Z}$ of course has a complicated cohomology as the covering $\mathcal{Z} \rightarrow \mathcal{Y}$ might be non-trivial, while $\mathcal{Y}$ is cohomologically the same as $\mathcal{X}$ and is nearly regular as a quotient of $\mathcal{Z}$.

\section{Construction of examples}

This section is devoted to the proof of Theorem 1.2 .

Let us first recall that if $E$ is a smooth genus 1 curve over a finite field $\mathbb{F}_{q}$, it is always an elliptic curve, which means that it always carries a $\mathbb{F}_{q}$-rational point. Furthermore one has

Claim 3.1. Given an elliptic curve $E / \mathbb{F}_{q}$, there is a finite field extension $\mathbb{F}_{q^{n}} \supset \mathbb{F}_{q}$ such that $\left|E\left(\mathbb{F}_{q^{n}}\right)\right|$ is not congruent to 1 modulo $q^{n}$.

Proof. By the trace formula, $\left|E\left(\mathbb{F}_{q^{n}}\right)\right|$ being congruent to 1 modulo $q^{n}$ for all $n \geq 1$ is equivalent to saying that the eigenvalues of $F^{n}$ acting on $H^{i}(\bar{E})$ lie in $q^{n} \cdot \overline{\mathbb{Z}}$ for all $n \geq 1$ and $i \geq 1$. By purity (which in dimension 1 is Weil's theorem), this is equivalent to saying that the eigenvalues of $F^{n}$ acting on $H^{1}(\bar{E})$ lie in $q^{n} \cdot \overline{\mathbb{Z}}$ for 
all $n \geq 1$. On the other hand, by duality, if $\lambda$ is an eigenvalue, then $\frac{q^{n}}{\lambda}$ is also an eigenvalue. It is then impossible that both $\lambda$ and $\frac{q^{n}}{\lambda}$ be $q^{n}$-divisible as algebraic integers.

We now construct the following scheme. Let us set $\mathcal{P}_{0}:=\mathbb{P}^{2}$ over $R_{0}:=\mathbb{Z}_{p}$ or over $\mathbb{F}_{p}[[t]]$. Choose an elliptic curve $E_{0} \subset \mathcal{P}_{0} \otimes \mathbb{F}_{p}=\mathbb{P}_{\mathbb{F}_{p}}^{2}$ defined over $\mathbb{F}_{p}$. Let $k \supset \mathbb{F}_{p}$ be a finite field extension such that $\left|E_{0}(k)\right|$ is not $k$-divisible (Claim 3.1). Set $E:=E_{0} \otimes_{\mathbb{F}_{p}} k, \mathcal{P}:=\mathcal{P}_{0} \otimes_{R_{0}} R$, with $R=W(k)$ or $\mathbb{F}_{q}[[t]]$, and $K=\operatorname{Frac}(R)$. Choose a smooth projective curve $\mathcal{C} \subset \mathcal{P}$ over $R$, of degree $\geq 4$, such that $C:=\mathcal{C} \otimes k$ is transversal to $E$. Define $\Sigma=E \cap C \subset E$ to be the 0-dimensional intersection subscheme. It has degree $\geq 12$, thus in particular $>9$. Let $b: \mathcal{Y} \rightarrow \mathcal{P}$ be the blow up of $\Sigma \subset \mathcal{P}$. We denote by $P_{\Sigma}$ the exceptional locus (which is a trivial $\mathbb{P}^{2}$ bundle over $\Sigma$ ), by $Y$ the strict transform of $\mathbb{P}_{k}^{2}$, and we still denote by $E \subset Y$ the strict transform of the elliptic curve. So one has the following diagram:

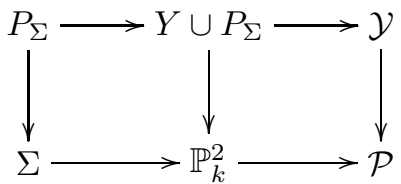

Then the conormal bundle $N_{E / \mathcal{Y}}^{\vee}$ of $E$ in $\mathcal{Y}$ is an extension of the conormal bundle $N_{E / Y}^{\vee}$ of $E$ in $Y$ by the restriction to $E$ of the conormal bundle $N_{Y / \mathcal{Y}}^{\vee}$ of $Y$ in $\mathcal{Y}$, both ample line bundles on $E$ by the condition on the degree of $\Sigma$.

Let $I \subset \mathcal{O}_{\mathcal{Y}}$ be the ideal sheaf of $E$. For a coherent sheaf $\mathcal{F}$ on $\mathcal{Y}$, we denote by $I^{n} / I^{n+1} \cdot \mathcal{F}$ the image of $I^{n} / I^{n+1} \otimes_{\mathcal{O}_{\mathcal{Y}}} \mathcal{F}$ in $\mathcal{F}$, where $n \in \mathbb{N}$.

Claim 3.2. For every coherent sheaf $\mathcal{F}$ on $\mathcal{Y}$, one has $H^{1}\left(E, I^{n} / I^{n+1} \cdot \mathcal{F}\right)=0$ for all $n \in \mathbb{N}$ large enough.

Proof. Since by definition one has a surjection $I^{n} / I^{n+1} \otimes_{\mathcal{O}_{\mathcal{y}}} \mathcal{F} \rightarrow I^{n} / I^{n+1} \cdot \mathcal{F}$, it is enough to show $H^{1}\left(E, I^{n} / I^{n+1} \otimes_{\mathcal{O}} \mathcal{F}\right)=0$ for $n$ large enough. As $I^{n} / I^{n+1}$ is locally free, $I^{n} / I^{n+1} \otimes_{\mathcal{O}_{\mathcal{Y}}} \mathcal{F}$ is an extension of $I^{n} / I^{n+1} \otimes_{\mathcal{O}_{\mathcal{Y}}} \mathcal{F}_{0}$ by $I^{n} / I^{n+1} \otimes_{\mathcal{O}_{\mathcal{Y}}} \mathcal{T}$, where $\mathcal{T} \subset \mathcal{F}$ is the maximal torsion subsheaf and $\mathcal{F}_{0}=\mathcal{F} / \mathcal{T}$ is locally free. Because $H^{1}\left(E, I^{n} / I^{n+1} \otimes_{\mathcal{O}} \mathcal{T} \mathcal{T}\right)=0$, we may assume that $\mathcal{F}$ is locally free. As $I^{n} / I^{n+1}$ is a locally free filtered sheaf, with associated graded a sum of ample line bundles of strictly increasing degree as $n$ grows, we have $H^{1}\left(E, \operatorname{gr}\left(I^{n} / I^{n+1}\right) \otimes_{\mathcal{O}} \mathcal{F}\right)=0$ for $n$ large enough, and thus $H^{1}\left(E, I^{n} / I^{n+1} \otimes_{\mathcal{O}} \mathcal{F}\right)=0$ as well.

Artin's contraction criterion [1, Theorem 6.2] applied to $E \rightarrow \operatorname{Spec}(k)$, together with Artin's existence theorem [1, Theorem 3.1], show the existence of a contraction

$$
a_{1}: \mathcal{Y} \rightarrow \mathcal{X}_{1}
$$

where $\mathcal{X}_{1}$ is an algebraic space over $R,\left.a_{1}\right|_{\mathcal{Y} \backslash E}$ is an isomorphism and $a_{1}(E)=$ $\operatorname{Spec}(k)$. Let $\mathcal{X} \stackrel{\nu}{\rightarrow} \mathcal{X}_{1}$ be the normalization of $\mathcal{X}_{1}$ in $K(\mathcal{Y})=K(\mathcal{P})$. This is a normal algebraic space over $R$. One has a diagram

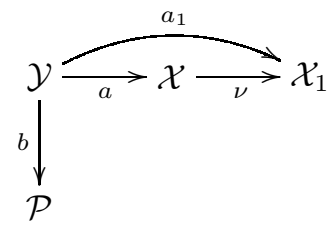


Claim 3.3. $|\mathcal{X}(k)|$ is not congruent to 1 modulo $|k|$.

Proof. Recall $a_{1}(E)$ is a rational point of $\mathcal{X}_{1}$. By [9, Theorem 1.1] (or by a simple computation in this case), $|\mathcal{Y}(k)|$ is congruent to 1 modulo $|k|$. By Claim 3.1 and the choice of $E,\left|\mathcal{X}_{1}(k)\right|$ is not congruent to 1 modulo $|k|$. On the other hand, as the fibers of $a_{1}$ are absolutely irreducible, $\nu$ has to be a homeomorphism. Thus $|\mathcal{X}(k)|=\left|\mathcal{X}_{1}(k)\right|$. This finishes the proof.

In order to finish the proof of Theorem [1.2, it remains to show

Claim 3.4. $\mathcal{X} \rightarrow \operatorname{Spec}(R)$ is a model of $X=\mathbb{P}^{2} / K$.

Proof. We have to show that $\mathcal{X} \rightarrow \operatorname{Spec}(R)$ is a flat projective morphism. Since $\mathcal{X}$ is integral and $\operatorname{Spec}(R)$ is regular of dimension 1, then [12, IV Proposition 14.3.8] allows us to conclude that $\mathcal{X} / R$ is flat. Thus we just have to show that $\mathcal{X} / R$ is projective. To this aim, we want a line bundle to descend from $\mathcal{Y}$ to an ample line bundle on $\mathcal{X}$. Recall $P_{\Sigma}=b^{-1}(\Sigma)$. Let us define the line bundle $\mathcal{M}:=$ $b^{*} \mathcal{O}_{\mathcal{P}}(\mathcal{C})\left(-P_{\Sigma}\right)$ on $\mathcal{Y}$. By definition, one has

$$
\left.\mathcal{M}\right|_{E} \cong \mathcal{O}_{E} .
$$

Claim 3.5. The line bundle $\mathcal{M}$ descends to $\mathcal{X}$, that is, there is a line bundle $\mathcal{L}$ on $\mathcal{X}$ with $a^{*} \mathcal{L}=\mathcal{M}$.

Proof of Claim 3.5. The proper morphism of algebraic spaces $a: \mathcal{Y} \rightarrow \mathcal{X}$, with $a_{*} \mathcal{O}_{\mathcal{Y}}=\mathcal{O}_{\mathcal{X}}$, has the property that $a^{-1} a(E)=E$ set-theoretically, that $\left.a\right|_{\mathcal{Y} \backslash E}$ : $\mathcal{Y} \backslash E \rightarrow \mathcal{X} \backslash a(E)$ is an isomorphism, and that $H^{1}\left(E, I^{n} / I^{n+1}\right)=0$ for $n \geq 1$. So Keel's theorem [13, Lemma 1.10] asserts that some positive power $\mathcal{M}^{\otimes r}$ descends to $\mathcal{X}$ if the following condition is fulfilled:

$$
\begin{gathered}
\forall m>0, \exists r(m)>0 \text { s.t }\left.\mathcal{M}^{\otimes r(m)}\right|_{E_{m}} \text { descends to } a\left(E_{m}\right), \\
\text { where } E_{m}:=\operatorname{Spec}\left(\mathcal{O}_{\mathcal{Y}} / I^{m+1}\right) .
\end{gathered}
$$

So we just have to check that (3.4) is fulfilled with $r=1$ in our situation. The scheme $a\left(E_{m}\right)$ has Krull dimension 0. Thus by Hilbert's Theorem 90 (see, e.g. 14, Corollary 11.6]) one has

$$
\operatorname{Pic}\left(a\left(E_{m}\right)\right)=0 .
$$

We conclude that to check (3.4) is equivalent to checking that $\left.\mathcal{M}^{\otimes r(m)}\right|_{E_{m}} \cong \mathcal{O}_{E_{m}}$ for some positive power $r(m)$. In fact one has

$$
\left.\mathcal{M}\right|_{E_{m}} \cong \mathcal{O}_{E_{m}} \forall m \geq 1 .
$$

For $m=1$, this is (3.3). We argue by induction and assume that for $m>1$, we have a trivializing section $s_{m}:\left.\mathcal{O}_{E_{m}} \cong \mathcal{M}\right|_{E_{m}}$. We want to show that it lifts to a trivializing section $s_{m+1}:\left.\mathcal{O}_{E_{m+1}} \stackrel{\cong}{\mathcal{M}}\right|_{E_{m+1}}$.

One has an exact sequence

$$
0 \rightarrow I^{m+1} /\left.\left.I^{m+2} \rightarrow \mathcal{M}\right|_{E_{m+1}} \rightarrow \mathcal{M}\right|_{E_{m}} \rightarrow 0 .
$$

Since $H^{1}\left(E, I^{m+1} / I^{m+2}\right)=0$, as $m \geq 0$, the trivializing section of $s_{m}: \mathcal{O}_{E_{m}} \cong$ $\left.\mathcal{M}\right|_{E_{m}}$ lifts to a section $s_{m+1}:\left.\mathcal{O}_{E_{m+1}} \rightarrow \mathcal{M}\right|_{E_{m+1}}$, and likewise, its inverse $t_{m}$ : 
$\left.\mathcal{M}\right|_{E_{m}} \stackrel{\cong}{\longrightarrow} \mathcal{O}_{E_{m}}$ lifts to $t_{m+1}:\left.\mathcal{M}\right|_{E_{m+1}} \rightarrow \mathcal{O}_{E_{m+1}}$. The composite $t_{m+1} \circ s_{m+1}:$ $\mathcal{O}_{E_{m+1}} \rightarrow \mathcal{O}_{E_{m+1}}$ lifts the identity of $\mathcal{O}_{E_{m}}$. Therefore it is invertible. This shows that $s_{m+1}$ trivializes. The proof of Keel's theorem (see (2) after [13, (1.10.1)]) then shows that one can take $r=1$.

In order the finish the proof of Claim 3.4, it remains to see that $\mathcal{L}$ on $\mathcal{X}$ is ample. We first show the following claim.

Claim 3.6. $\left.\mathcal{L}\right|_{\mathcal{X} \otimes k}$ is an ample line bundle on $\mathcal{X} \otimes k$.

Proof. We first show that $\left.\mathcal{M}\right|_{\mathcal{Y} \otimes k}$ is nef and big. In fact, we prove a more precise property: for any irreducible curve $\Gamma$ on $\mathcal{Y} \otimes k$, one has $\left.\mathcal{M}\right|_{\mathcal{Y} \otimes k} \cdot \Gamma \geq 0$, and the equality holds if and only if $\Gamma=E$. By construction, $\mathcal{Y} \otimes k=P_{\Sigma} \cup Y$ and each component over $\bar{k}$ of $P_{\Sigma}$ is isomorphic to $\mathbb{P}_{\bar{k}}^{2}$. Since the restriction of $\mathcal{M}$ on every component of $P_{\Sigma}$ is isomorphic to $\mathcal{O}(1)$, we can assume $\Gamma \subset Y$. The embedding $E \subset Y$ is a section of the line bundle $\left.b\right|_{Y} ^{*} \mathcal{O}(3)\left(-E_{\Sigma}\right)$, where $E_{\Sigma}=P_{\Sigma} \cap Y$. There is also a large enough $n$, such that $H=\left.b\right|_{Y} ^{*} \mathcal{O}(n)\left(-E_{\Sigma}\right)$ is ample. So $\left.\mathcal{M}\right|_{Y}=$ $\left.b\right|_{Y} ^{*} \mathcal{O}(C)\left(-E_{\Sigma}\right) \equiv_{\mathbb{Q}} e_{0} E+e_{1} H$, where $0<e_{0}, e_{1}<1$ and $e_{0}+e_{1}=1$. From this, we easily see that $\left.\mathcal{M}\right|_{Y} \cdot \Gamma>0$, when $\Gamma \subset Y$ and $\Gamma \neq E$. The above argument also shows the bigness of $\left.\mathcal{M}\right|_{\mathcal{Y} \otimes k}$ : on $P_{\Sigma}$, it is ample; and on $Y$, it is a convex combination of an effective divisor and of an ample divisor.

Since $a^{*}(\mathcal{L})=\mathcal{M}$, the nefness and bigness of $\left.\mathcal{M}\right|_{\mathcal{Y} \otimes k}$ imply that the same properties hold for $\left.\mathcal{L}\right|_{\mathcal{X} \otimes k}$. So $\left.\mathcal{L}\right|_{\mathcal{X} \otimes k}$ is semiample by [13, Corollary 0.3]. Furthermore, the more precise property we proved above for $\left.\mathcal{M}\right|_{\mathcal{Y} \otimes k}$ implies that the intersection of $\left.\mathcal{L}\right|_{\mathcal{X} \otimes k}$ with any curve on $\mathcal{X} \otimes k$ is positive; thus we conclude $\left.\mathcal{L}\right|_{\mathcal{X} \otimes k}$ is ample.

So by the Serre vanishing theorem, for sufficiently large $m, H^{1}\left(\mathcal{X} \otimes k,\left.\mathcal{L}\right|_{\mathcal{X} \otimes k} ^{\otimes m}\right)=$ 0 . Base change implies $H^{1}\left(\mathcal{X}, \mathcal{L}^{\otimes m}\right) \otimes k=0$ ([12, III Theorem 7.7.5]); thus by Nakayama's lemma, one has

$$
H^{1}\left(\mathcal{X}, \mathcal{L}^{\otimes m}\right)=0 \text { for } m \text { large enough. }
$$

As $\mathcal{L}$ is invertible, multiplication $\mathcal{L}^{\otimes m} \stackrel{\pi}{\rightarrow} \mathcal{L}^{\otimes m}$ by the uniformizer $\pi$ is injective, with quotient $\left.\mathcal{L}\right|_{\mathcal{X} \otimes k} ^{\otimes m}$. Thus (3.8) implies surjectivity

$$
H^{0}\left(\mathcal{X}, \mathcal{L}^{\otimes m}\right) \rightarrow H^{0}\left(\mathcal{X} \otimes k,\left.\mathcal{L}\right|_{\mathcal{X} \otimes k} ^{\otimes m}\right)
$$

for $m$ large enough. Thus $H^{0}\left(\mathcal{X}, \mathcal{L}^{\otimes m}\right)$ is a free $R$-module, and the linear system $H^{0}\left(\mathcal{X}, \mathcal{L}^{\otimes m}\right)$ maps without base points $\mathcal{X}$ to $\mathbb{P}_{R}^{N}$, with $N+1=\operatorname{rank}_{R} H^{0}\left(\mathcal{X}, \mathcal{L}^{\otimes m}\right)$. As it embeds $\mathcal{X} \otimes k$, it embeds $\mathcal{X}$ as well. This finishes the proof.

\section{REMARKS}

Remark 4.1. In Theorem 1.1, if $X / K$ has dimension 1 , which means concretely if $X / K=\mathbb{P}^{1} / K$, then any normal model $\mathcal{X} / R$ satisfies the congruence $|\mathcal{X}(k)| \equiv 1$ modulo $|k|$. Thus the examples of Theorem 1.2 have the smallest possible dimension.

Proof. Indeed, using (2.1), the only thing to check is that $H^{1}(\bar{A})$, which is equal to $H^{1}\left(\mathcal{X}^{u}\right)$, injects via $\sigma^{*}$ into $H^{1}(\bar{B})=H^{1}\left(\mathcal{Y}^{u}\right)$. Here $A:=\mathcal{X} \otimes_{R} k$. Let us denote 
by $\mathcal{X}^{\prime}$ the normalization of $\mathcal{X}$ in $K(\mathcal{Y})$, with factorization

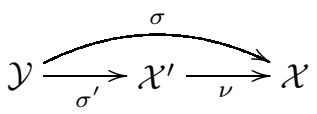

and set $A^{\prime}:=A \times \mathcal{X} \mathcal{X}^{\prime}$. Then $\sigma^{\prime}$ induces an isomorphism $K\left(\mathcal{X}^{\prime}\right) \cong K(\mathcal{Y})$. Furthermore, $\mathcal{X}^{\prime} \stackrel{\nu}{\rightarrow} \mathcal{X}$ and and $A^{\prime} \stackrel{\left.\nu\right|_{A}}{\longrightarrow} A$ are homeomorphisms. Thus $H^{1}\left(\mathcal{X}^{u}\right)=$ $H^{1}(\bar{A}) \stackrel{\nu^{*}}{\longrightarrow} H^{1}\left(\left(\mathcal{X}^{\prime}\right)^{u}\right)=H^{1}\left(\bar{A}^{\prime}\right)$ is an isomorphism. On the other hand, since $\sigma_{*}^{\prime} \mathbb{Q}_{\ell}=\mathbb{Q}_{\ell}$, the Leray spectral sequence for $\sigma^{\prime}$ applied to $H^{1}\left(\mathcal{Y}^{u}\right)$ yields an inclusion $H^{1}\left(\left(\mathcal{X}^{\prime}\right)^{u}\right)=H^{1}\left(\bar{A}^{\prime}\right) \stackrel{\text { inj }}{\longrightarrow} H^{1}\left(\mathcal{Y}^{u}\right)=H^{1}(\bar{B})$. This finishes the proof.

Remark 4.2. We generalize Remark 4.1 to the higher dimensional case in the following form. Let $X$ be a smooth projective variety defined over $K$ and let $\mathcal{X} / R$ be a model over $R$. Let us use the notation of (2.1). We set $A=\mathcal{X} \otimes_{R} k, B=\mathcal{Y} \otimes_{R} k$. If the assumptions of Theorem 1.1 are fulfilled, that is, if $\ell$-adic cohomology $H^{i}(\bar{X})$ is supported in codimension $\geq 1$ for all $i \geq 1$, and if in addition

$$
\sigma^{*}: H^{i}\left(\mathcal{X}^{u}\right)=H^{i}(\bar{A}) \rightarrow H^{i}\left(\mathcal{Y}^{u}\right)=H^{i}(\bar{B})
$$

is injective for all $i \geq 0$, then one has

$$
|\mathcal{X}(k)| \equiv 1 \text { modulo }|k| \text {. }
$$

Indeed, the exact sequence (2.2) together with Claim 2.1 and Claim 2.2 show that under the assumptions of Theorem 1.1 one has

$$
\text { eigenvalues of } F \text { acting on } H^{i}(\bar{B}) \in q \cdot \overline{\mathbb{Z}} \forall i \geq 1 \text {. }
$$

As $\sigma^{*}$ in (4.2) is equivariant (which of course we already used in the proof of Theorem 1.1), we conclude

$$
\text { eigenvalues of } F \text { acting on } H^{i}(\bar{A}) \in q \cdot \overline{\mathbb{Z}} \forall i \geq 1 \text {. }
$$

Since $H^{i}\left(\mathcal{Y}^{u}\right)=H^{i}\left(\mathcal{Z}^{u}\right)^{G} \subset H^{i}\left(\mathcal{Z}^{u}\right)$, injectivity of $\sigma^{*}$ in (4.2) is equivalent to injectivity of

$$
\tau^{*} \circ \sigma^{*}: H^{i}\left(\mathcal{X}^{u}\right) \rightarrow H^{i}\left(\mathcal{Z}^{u}\right) .
$$

One may ask the following question:

Question 4.3. Let $\mathcal{X}$ be an integral $R$-scheme. What are the types of singularities of $\mathcal{X}$ which force the following: for any alteration $\pi: \mathcal{Y} \rightarrow \mathcal{X}$ in the sense of de Jong, that is, $\pi$ is proper, dominant with $K(\mathcal{X}) \subset K(\mathcal{Y})$ finite, and with $\mathcal{Y}$ regular, one has that the induced map $\pi^{*}: H_{c}^{i}(\mathcal{X}) \rightarrow H_{c}^{i}(\mathcal{Y})$ on compactly supported $\ell$-adic cohomology is injective?

P. Berthelot ([3]) observes that if $\pi$ is generically étale, that is, if $K(\mathcal{X}) \subset K(\mathcal{Y})$ is separable and $\mathcal{X}$ is regular, then purity as in 11 implies immediate injectivity of $\pi^{*}$. Of course, from the viewpoint of point counting, since regularity of $\mathcal{X}$ is the assumption under which the main result of 9 ] was shown, this does not bring any new information. However, this, together with Theorem 1.2 of this note, suggests singling out a good definition of mild singularities for $\mathcal{X}$ which would force injectivity of $\pi^{*}$. There is the extra problem of separability of $K(\mathcal{X}) \subset K(\mathcal{Y})$. It would be nice not to have it as an assumption. Theorem 1.1 perhaps suggests that this is not the main point. 
Remark 4.4. We can lower the level of difficulty of Question 4.3 by considering varieties $A$ defined over finite field $k$, or even a perfect field. In this situation, a notion of Witt-rational singularities was introduced in [4, which echoes the notion of rational singularities in characteristic zero and which relies on the slope theorem [2. Theorem 1.1] in Berthelot's rigid cohomology. Working $\ell$-adically, the corresponding notion may be: let $A$ be a variety defined over a finite field $k$. Then $A$ has $\ell$-adic rational singularities if for any alteration $\pi: B \rightarrow A$, the induced map $\pi^{*}: H_{c}^{i}(\bar{A}) \rightarrow H_{c}^{i}(\bar{B})$ is injective on the maximal subspace $H_{c}^{i}(\bar{A})^{<1}$ of $H_{c}^{i}(\bar{A})$, which is invariant under the geometric Frobenius $F$, and on which $F$ acts with eigenvalues not in $q \cdot \overline{\mathbb{Z}}$. Such a definition will force the point counting to work as on smooth $A$. For example, 4, Theorem 1.1] would work similarly, with "Wittrational singularities" replaced by $\ell$-adic rational singularities. But somehow, this is of restricted interest: the beauty of rational singularities in chararcteristic 0 is that due to their definition via coherent cohomology, one can understand geometrically well what they are. A definition directly via étale cohomology somehow does not give such an immediate geometric picture.

\section{ACKNOWLEDGEMENTS}

We thank Johan de Jong for his interest, and Dan Abramovich for a careful reading which helped us in clarifying the exposition.

\section{REFERENCES}

[1] Artin, M.: Algebraization of Formal Moduli: II. Existence of Modifications, Ann. of Math. (2) 91 (1970), 88-135. MR0260747 (41:5370)

[2] Berthelot, P., Bloch, S., Esnault, H.: On Witt vector cohomology for singular varieties, Compositio Mathematica 143 (2007), 363-392. MR2309991 (2008b:14023)

[3] Berthelot, P.: letter to Hélène Esnault dated April 23. 2007.

[4] Blickle, M., Esnault, H.: Rational singularities and rational points, preprint 2006, 12 pages, appears in the volume dedicated to F. Bogomolov, Pure and Applied Math. Quarterly.

[5] de Jong, A. J.: Smoothness, semi-stability and alterations, Publ. Math. IHES 83 (1996), 51-93. MR.1423020 (98e:14011)

[6] de Jong, A. J.: Families of curves and alterations, Ann. Inst. Fourier 47 no. 2 (1997), 599-621. MR.1450427 (98f:14019)

[7] Deligne, P.: Théorème d'intégralité, Appendix to Katz, N.: Le niveau de la cohomologie des intersections complètes, Exposé XXI in SGA 7, Lect. Notes Math. vol. 340, 363-400, Berlin, Heidelberg, New York, Springer, 1973. MR0354657(50:7135)

[8] Deligne, P.: La conjecture de Weil, II. Publ. Math. IHES 52 (1981), 137-252. MR601520 (83c:14017)

[9] Esnault, H.: Deligne's integrality theorem in unequal characteristic and rational points over finite fields, with an appendix with P. Deligne, Ann. of Math. 164 (2006), 715-730. MR2247971 (2007g:14019)

[10] Esnault, H.: Coniveau over p-adic fields and points over finite fields, C. R. Acad. Sci. Paris, Sér. I 345 (2007), 73-76. MR2343555

[11] Fujiwara, K.: A Proof of the Absolute Purity Conjecture (after Gabber), in Algebraic Geometry 2000, Azumino, Advanced Studies in Pure Mathematics 36 (2002), Mathematical Society of Japan, 153-183. MR1971516 (2004d:14015)

[12] Grothendieck, A.: Éléments de Géométrie Algébrique (EGA): III (2): Étude cohomologique des faisceaux cohérents, Publ. Math. IHES 17 (1963) IV (3): Études locales des schémas et des morphismes de schémas, Publ. Math. IHES 28 (1966). 
[13] Keel, S.: Basepoint freeness for nef and big line bundles in positive characteristic, Ann. of Math. (2) 149 (1999), no. 1, 253-286. MR1680559 (2000j:14011)

[14] Milne, J.: Lectures on Étale Cohomology, v2 02, August 9 (1998), http://www.jmilne. org/math/.

Abteilung von Mathematik, Universität Duisburg-Essen, 45117 Essen, Germany

E-mail address: esnault@uni-due.de

Department of Mathematics, Princeton University, Princeton, New Jersey 08544

E-mail address: chenyang@math.princeton.edu

Current address: School of Mathematics, Institute for Advanced Study, Einstein Drive, Princeton, New Jersey 08540

E-mail address: chenyang@ias.edu 